\title{
Die Pollenkammer als Studierstube
}

Die Beschwerden während Provokationstests in der Pollenkammer korrelieren gut mit den Symptomen, die bei natürlicher Pollenbelastung auftreten. Texanische Forscher haben dabei drei Endotypen der allergischen Rhinitis ausgemacht.

D rovokationstests in einer Pollenkammer haben für Studienzwecke u. a. den Vorteil, dass standardisierte Belastungen mit klar definierten Pollensorten vorgenommen werden können. Es ist aber noch wenig erforscht, wie gut die dabei evozierten Symptome mit jenen unter natürlicher Pollenbelastung korrelieren.

Robert Jacobs und Kollegen haben sich dieser Frage angenommen. Die Forscher bedienten sich dabei des ortsüblichen natürlichen Pollenlieferanten, des Dreiblättrigen Traubenkrauts (Ambrosia trifida). Für die Belastungstests in der Pollenkammer, die außerhalb der Pollensaison stattfanden, benutzten sie Pollen des Beifußblättrigen Traubenkrauts (Ambrosia artemisiifolia). 31 auf Traubenkraut sensitive Freiwillige ließen sich auf die Versuche ein. Sie führten wäh- rend der Hauptblütezeit im September und Oktober 2010 während 15 Tagen über ihre Beschwerden Buch. 26 von ihnen rückten dann im November in die Pollenkammer ein, wo sie - sofern sie durchhielten - bei vier je dreistündigen Besuchen einer Pollenbelastung ausgesetzt wurden.

Zwölf der 26 Pollenkammer-Probanden mussten vorzeitig das Taschentuch werfen, 14 hielten alle vier Sitzungen durch. Die erste Gruppe wurde als rasch und hoch reaktiv eingestuft (RHR), die zweite als langsam und niedrig reagierend (LNR). Generell lagen die Symptomenscores in der Pollenkammer etwas unter jenen in freier Wildbahn, im Wesentlichen aber waren sie vergleichbar. Augenjucken, -tränen und -rötung, verstopfte und juckende Nase - alle diese Beschwerden korrelierten trotz unterschiedlicher Pollenarten signifikant $(\mathrm{p}<0,01)$. Ausnahmen bildeten nur das Niesen und die Nasensekretion.

Hinsichtlich der Ergebnisse in der Kammer und in der Natur ließen sich drei Endotypen ausmachen. Typ I war in der Pollenkammer und unter natürlicher Pollenlast dem Reaktionsmodus LNR zuzuordnen. Für Typ II galt Gleiches, aber im Modus RHR. Typ III schließlich reagierte als LNR in der Kammer und als RHR an der frischen Luft.

Fazit: Pollenkammern für Provokationstests zur allergischen Rhinokonjunktivitis sind geeignet, unterschiedliche Reaktionstypen zu identifizieren. Dabei kann man offenbar Kreuzreaktionen nutzen. Den Endotypen I bis III liegen womöglich Unterschiede in der Pathogenese zugrunde, die sich auch auf die Therapie auswirken könnten.

Dr. Robert Bublak

Jacobs RL et al. Responses to ragweed pollen in a pollen challenge chamber versus seasonal exposure identifies allergic rhinoconjunctivitis endotypes. J Allergy Clin Immunol 2012, 130: 122-7.e8

\section{ACHTUNG:}

\section{Hier muss der Dummy durch eine Anzeige ersetzt werden !!}

